\title{
Amphiphilic Polymer Platforms: Surface Engineering of Films for Marine Antibiofouling
}

\author{
Giancarlo Galli, Elisa Martinelli \\ Dipartimento di Chimica e Chimica Industriale and UdR Pisa INSTM, Università di \\ Pisa, 56124 Pisa, Italy
}

Corresponding authors

Email: giancarlo.galli@unipi.it, elimart79@dcci.unipi.it

Giancarlo Galli is a professor of Industrial Chemistry at the University of Pisa. He spent periods of time at the University of Liverpool, CNRS-Orleans, Cornell University, Chalmers Technical University and Tokyo Institute of Technology. His research interests are on the chemistry, characterisation and application of low-impact and sustainable polymeric materials for the environment, biology and medicine, information technology and optoelectronics. He was Chairman of the European Polymer Federation (2012-2013). He is a member of the Editorial Board of Macromolecular Rapid Communications and Macromolecular Chemistry and Physics.

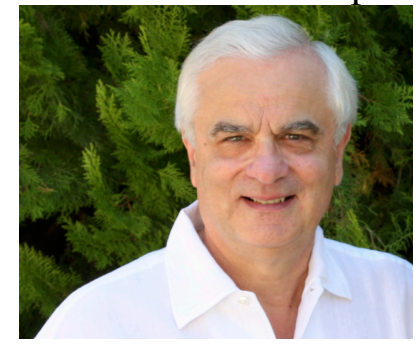

Elisa Martinelli received her $\mathrm{PhD}$ in Chemical Sciences in 2004 at the University of Pisa. In 2008-2014 she held post-doc positions at the Department of Chemistry and Industrial Chemistry (University of Pisa) where she is now a research associate and lecturer. She spent some periods of time doing research abroad, including those at Ivoclar-Vivadent AG Schaan, the School of Biosciences, University of Birmingham, and the School of Marine Science and Technology, University of Newcastle.

Her research interests are mainly focused on the synthesis and characterization of polymers and nanostructured materials for innovative and advanced applications with low environmental impact.

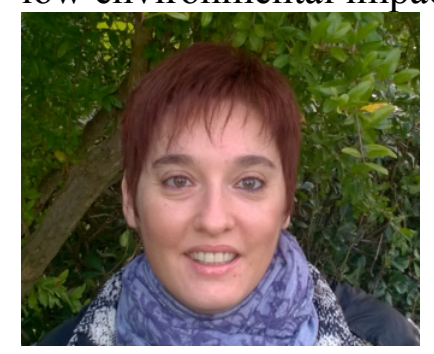




\begin{abstract}
A range of amphiphilic polymers with diverse macromolecular architectures has been developed and incorporated into films and coatings with potential in marine antibiofouling application without resorting to addition of currently used biocidal, toxic agents. Novel 'green' chemical technologies employ different building blocks to endow the polymer film with surface activity, functionality, structure, and reconstruction according to the outer environment as a result of a tailored amphiphilic character of the polymer platform. We emphasise how these features can interplay and add synergistically to effect antifouling and fouling-release against common, widespread marine micro- and macro-fouling organisms.
\end{abstract}

\title{
1. Introduction
}

Materials surfaces and interfaces are always prone to the absorption of molecules, proteins, cells and organisms depending on the diverse application contexts. ${ }^{[1]}$ Adhesion of these (bio)foulers occurs by a number of mechanisms involving chemical bonding, physical interaction and adsorption, mechanical interlocking and surface rearrangement. ${ }^{[2]}$ Such coverage and accumulation of foreign entities heavily impacts on the surface and interface properties of the material that rapidly loses its original performance. Specifically, marine biofouling consists of the unwanted and uncontrolled adhesion and proliferation of a vast range of marine organisms on a surface immersed in seawater. ${ }^{[3]}$ According to a much quoted view, marine biofouling is a complex process consisting of a number of key stages of growth (Figure 1). ${ }^{[4,5]}$ Within minutes of immersion, a pristine surface becomes conditioned through the adsorption of macromolecules, e.g. proteins, polysaccharides and glycoproteins. Bacteria colonise the surface and create a biofilm matrix. Initially this stage is reversible, as planktonic bacteria adhere weakly to the surface by non-covalent van der Waals or electrostatic interactions. Then bacteria irreversibly adhere to the surface through the secretion of a cellular appendix and exopolymers. The formation of such a microbial film favours the settlement of multicellular species, e.g. spores of algae, generally known as microfoulers. This then paves the way for the settlement of macrofoulers, such as barnacles, bryozoans and tubeworms, and further algal growth. However, this successional model holds true only for some fouling species and the 
formation of a biofilm is not always necessary for the colonisation of macrofoulers. ${ }^{[6]}$ In fact spores of the macroalga Ulva linza ${ }^{[7]}$ and larvae of the barnacle Balanus amphitrite $^{[8]}$ can settle on clean surfaces within minutes after immersion.

Biological settlement has detrimental effects on equipment and structures employed in the maritime industry (Figure 1), such as ships and leisure crafts (T\&S), heat exchanger tubes and marine energy turbines (ME), harbour installations, oceanographic sensors (Sens), seawater filtration membranes and aquaculture systems (AQF) ${ }^{[4,8-12]}$ For shipping, the adverse effects include high frictional resistance, due to generated roughness, which leads in turn to an increase in fuel consumption and a reduction in cruising speed. ${ }^{[13,14]}$ In addition, hull cleaning, paint removal and repainting, and associated environmental compliance measures contribute to the cost of biofouling. ${ }^{[15]}$ Moreover, fouled ship hulls can transport invasive alien species in a non-native environment, thus causing problems to the ecosystem. ${ }^{[16,17]}$

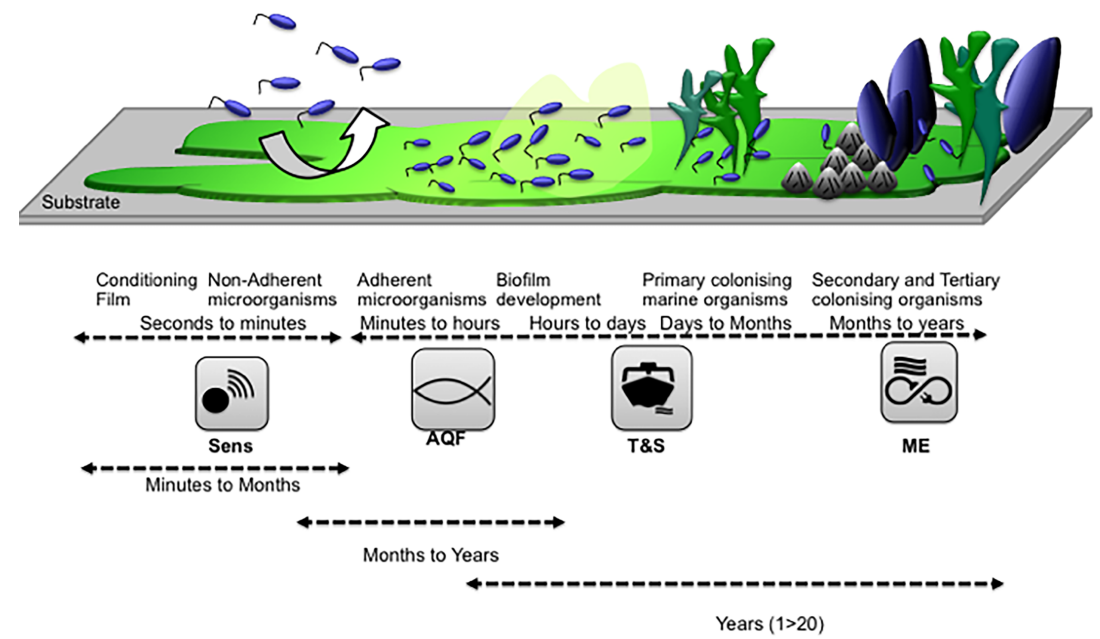

Figure 1. Schematic showing biofouling progression: the rate and degree of fouling impacting the maritime industry/activity and the timeframe over which antifouling solutions are needed to be effective. Reproduced with permission from Ref. ${ }^{[4]}$. Photo: MESTECH, as published in the JOT, V9N4, 2014.

Marine biofouling has been controlled for almost 50 years by the use of antifouling paints based on derivatives of tributyltin (TBT), a broad-spectrum biocide introduced in the marine coating industry in the late $1950 \mathrm{~s} .{ }^{[18]}$ TBT has been proved to be highly toxic toward non-target aquatic organisms and damage the marine 
environment. ${ }^{[19]}$ The negative effects of TBT forced many governments to restrict its use and its ban came in force in 2008. ${ }^{[20]}$ The current generation of commercial antifouling coatings is normally based on self-polishing copolymer paints (SPC), which contain high levels of copper (40-75 wt\%) and booster biocides, e.g. zinc and copper pyrithione. ${ }^{[21]}$ However, environment contamination due to leaching of copper from SPC coatings is also a global issue, as copper concentrations higher than $3.1 \mathrm{ppb}$ (the U.S. federal standard) affect various life stages of marine organisms including crustaceans, oysters, mussels and sea urchins. ${ }^{[22]}$ Due to these environmental concerns, biocidal coatings are becoming increasingly more regulated, creating a demand for high performance non-toxic alternatives. ${ }^{[1,23,24]}$

Being biofouling a natural nanoscale process, which involves interfacial interactions of the specific structural elements of the organism's adhesive with the outermost few nanometers of the substrates, its ultimate control may lie in modifying the properties of a surface at the nanoscale by means of novel chemistries for surface engineering. ${ }^{[25]}$ Diverse properties are relevant in determining the performance of coatings against marine organisms by an antifouling (AF) capacity, i.e. an initial prevention of biofoulers from attaching to a surface, and/or a fouling-release (FR) efficacy, i.e. an easy detachment under low shear stress of biofoulers possibly attached to a surface with a weak bond. Whilst it is arguably a combination of features that will eventually intervene in the overall performance of a coating, the development of a 'model' surface in which a single surface property is systematically changed, while keeping the others constant, is a tool to identify the influence of individual surface features on biofouling formation, settlement and removal. Although it is difficult to compare different driving forces for surface fouling in terms of their independent and cooperative effects in affecting adhesion on a quantitative level, ${ }^{[26]}$ comprehensive investigations would eventually lead to a knowledge-based, exploitable understanding for the development of 'practical' coatings for application to structures for the aquatic environment on a large industrial scale for different enduses.

Advances in the design of model and practical films for antifouling encompass the preparation of nanostructured surfaces according to top-down and bottom-up strategies. The former employ sophisticated technologies, such as photolithographic patterning, microcontact printing, hot pressing moulding and micromoulding in 
capillaries and their use is generally limited to model surfaces, quite often biomimetic surfaces, for exploring adhesion mechanisms. ${ }^{[27,28]}$ The latter strategies are based on various alternatives of supramolecular chemistry, self-assembly, phase-separation of polymers and chemical building blocks for the creation of coatings with tunable nanoscale surface properties. Lately a range of approaches has been investigated that span from bioinspired polymers incorporating bioactive molecules and enzymes ${ }^{\text {[29-31] }}$ to hydrophobic or hydrophilic self-assembling monolayers, ${ }^{[32-35]}$ hydrophobic fluoropolymer networks, ${ }^{[36-39]}$ antimicrobial quaternary ammonium polymers, ${ }^{[40,41]}$ self-stratifying polysiloxane hybrids, ${ }^{[29,42-45]}$ poly(2-methyl-2-oxazoline) $\mathrm{s}^{[46]}$ and polyethylene glycol hydrogels, ${ }^{[47,48]}$ xerogels,${ }^{[49-51]}$ zwitterionic polymers, ${ }^{[52]}$ layer-bylayer assemblies, ${ }^{[53,54]}$ nanocomposites containing nanofillers of varying nanoscale dimensions $^{[53-57]}$ and photocatalytic nanoparticles ${ }^{[21,58,59]}$, to self-polishing silyl ester polymers. ${ }^{[60]}$

The engineering of the surface and interface of amphiphilic polymers, which combine both hydrophilic and hydrophobic components in one chemical architecture, has attracted attention in the last decade and is currently conceived as one more promising strategy to implement special interface functions in a variety of industries and technologies. ${ }^{[61]}$ Most recent examples include the fields of medical implants and devices, ${ }^{[62]} \mathrm{drug}^{[63]}$ and gene ${ }^{[64]}$ delivery, texturing, ${ }^{[65]}$ petroleum recovery, ${ }^{[66]}$ and membrane separation processes. ${ }^{[67]}$ Amphiphilic polymers are regarded as especially liable materials to combat marine biofouling. ${ }^{[71-73]}$ Several such types of coatings have been commercialised, e.g. those of the brands Intersleek and Hempasil. ${ }^{[62-64]}$ The potential of amphiphilic polymers resides in the ability to provide a heterogeneous nanoscale mosaic chemical surface, where the coexistence of hydrophobic and hydrophilic domains can confuse organisms during settlement and adhesion. Different organisms in fact show different adhesion profiles and contrasting preferences. For example, two types of marine algae, viz. the macroalga $U$. linza and the unicellular diatom Navicula perminuta, have preferences for substrates with opposite wettability; ${ }^{[6]}$ while $N$. perminuta adheres weakly to hydrophilic surfaces, U. linza typically adheres more strongly to hydrophilic substrates. Whereas coatings with a rigorous, purely hydrophobic, or otherwise hydrophilic, nature display a limited antifouling activity, amphiphilic coatings having compositional control over hydrophobic and hydrophilic components can potentially exhibit a broader spectrum 
of efficacy against marine biofouling when the individual characteristics add synergistically to each other.

Herein we present recent advances in the field of novel amphiphilic polymer platforms for which macromolecular engineering of the surface properties helped to improve marine AF/FR performance. Selected examples of polymer films that were tested at least by laboratory assays against one or more of the marine species generally accepted as model organisms of either microfouling (bacteria and diatoms) or macrofouling (algae, barnacles and tubeworms) will be discussed. Emphasis was focused on the chemistry and architecture of the amphiphilic polymers, especially in relation to their response to the water environment. We identified the multifaceted, intertwined characters of (i) activity, (ii) functionality, (iii) structure and (iv) reconstruction of the film surface as major driving factors to improved performance and highlighted the role they play in combating marine biofouling. These may serve as general guidelines to develop novel amphiphilic polymers with improved marine AF/FR effectiveness.

\section{Amphiphilic surface-active polymers}

A surface-active polymer is a tailored material able to phase-segregate and selfassemble at the polymer-environment interface when incorporated into a more complex system so as to modify the surface properties of the entire system. Typical surface-active polymers are composed of low surface energy fluoropolymers. ${ }^{[74-76]}$

Polymers with liquid crystalline fluoroalkyl side chains were used to create highly non-polar hydrophobic surfaces, which were effective in releasing model macrofoulers $U$. linza and B. amphitrite. ${ }^{[36,37]}$ However, they had limitations in the inhibition of protein adsorption and promotion of diatom release. ${ }^{[36]}$ A new generation of amphiphilic surface-active (co)polymers was designed by combination of poly(ethylene glycol) (PEG) with a fluorinated component. PEG is known to resist cell adhesion and protein adsorption, owing to its low interfacial energy with water $\left(<5 \mathrm{mN} \mathrm{m}^{-1}\right)$. This makes the intramolecular forces between the biomacromolecules and the substratum thermodynamically unfavourable, the interfacial energy being sufficiently low. 
Amphiphilic copolymers have been used to prepare AF/FR films as either individual systems ${ }^{[77-81]}$ or dispersed within an elastomeric, supporting matrix. ${ }^{[68,82,83]}$ The amphiphilic surface-active copolymer and the elastomeric matrix are generally combined together according to one- and two-layer geometries. In a one-layer geometry the amphiphilic copolymer is dissolved with an elastomeric matrix and the solution is cast on a pre-treated solid support. During the solvent evaporation, phasesegregation of the low surface energy copolymer drives most of it to the polymer-air interface. In a two-layer geometry, the elastomer is solution cast to form a first, bottom thick layer $(>100-200 \mu \mathrm{m})$; a second, top thin layer $(<5-10 \mu \mathrm{m})$ consisting of the amphiphilic copolymer either alone or blended with the elastomer is then coated on top of the bottom layer. This latter geometry has the main advantage to enable an independent control of the bulk thickness and elastic modulus on the one hand, and the surface and interface properties on the other hand, by the use of a low amount (normally $<5-10 \mathrm{wt} \%$ ) of an amphiphilic surface-active polymer. In both film geometries the copolymer is physically dispersed in, not chemically linked to the elastomeric matrix. The matrices generally used for this purpose were poly(styrene- $b$ (ethylene-co-butylene)- $b$-styrene) (SEBS) and poly(dimethylsiloxane) (PDMS). While the elastomeric character of the SEBS-based coatings is inherent in the physically cross-linked structure of the block copolymer matrix, the elastomeric PDMS-based coatings are chemically cross-linked by a sol-gel curing reaction of the PDMS matrix. This requires a metal catalyst to occur, ordinarily Pt- and Sn-catalysts, or Bi-catalysts in a more eco-sustainable approach. ${ }^{[84,85]}$

\subsection{SEBS-based systems}

Initial work ${ }^{[68]}$ dealt with a poly(styrene-b-acrylic acid) block copolymer that was post-modified to attach amphiphilic PEGylated-perfluoroalkyl side chains (1, Figure 2). While the polystyrene block acted as a binder with the SEBS matrix, the PEGylated-perfluoroalkyl chains imparted amphiphilicity and environment-dependent response in surface chemistry to the entire coating. Both the settlement and removal of $U$. linza zoospores and sporelings (young plants) were found to be comparable with that of the PDMS standard. On the other hand, the removal of $N$. perminuta cells from the amphiphilic coatings was significantly higher with respect to the PDMS standard. This result was attributed to a reconstruction of the surface that became more 
hydrophilic after immersion in water, as a consequence of the flipping of the amphiphilic side chain in order to favour exposure of the hydrophilic PEGylated segment to contact with water (Figure 3).

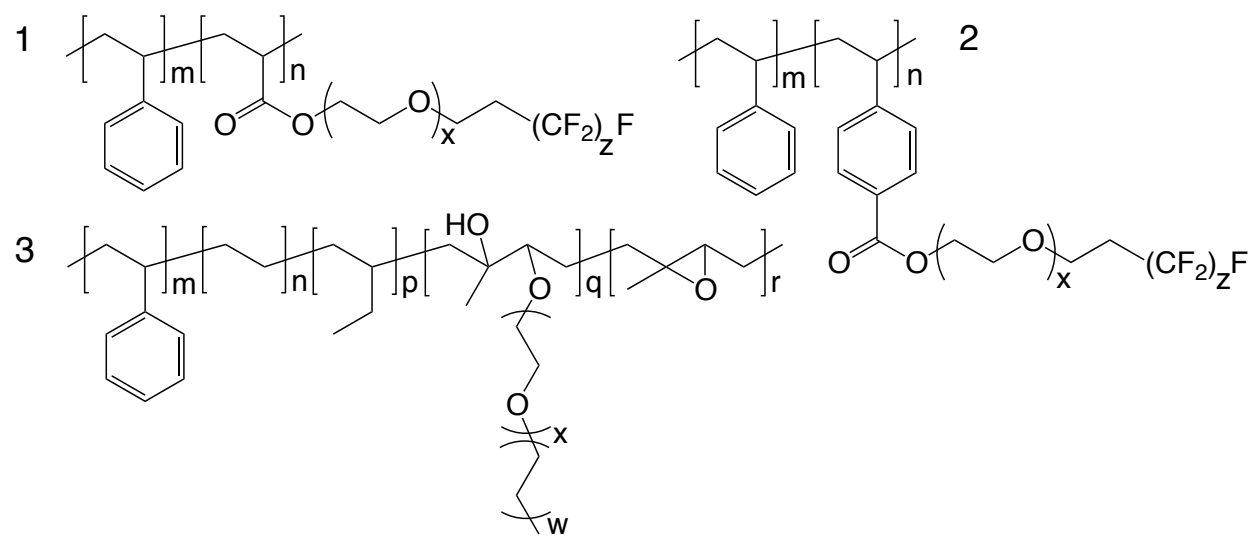

Figure 2. Illustrations of surface-active (co)polymers containing hydrophilic PEG segments, hydrophobic alkyl segments and hydrophobic/lipophobic fluorinated segments of varying length ( $\mathrm{x}, \mathrm{w}$ and $\mathrm{z}$, respectively) for introduction into SEBSbased systems. ${ }^{[68,86,87]}$

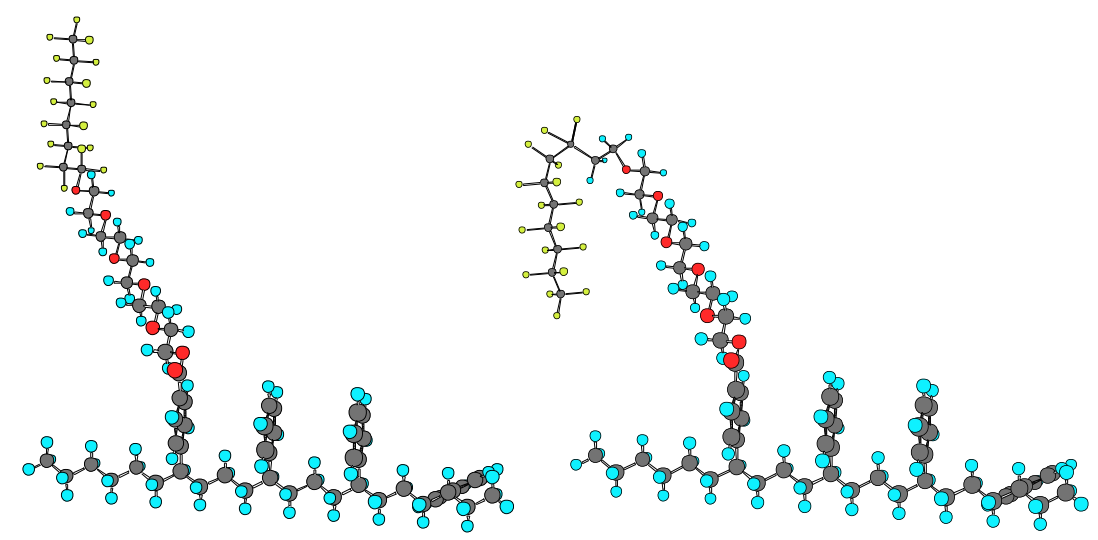

Figure 3. Schematic of a proposed surface reconstruction by flipping of PEGylatedperfluoroalkyl side chains upon immersion of the surface in water: orientation of side chains in air (left) and in water (right). Redrawn after Ref. ${ }^{[68]}$.

Later on, diblock copolymers of polystyrene and polystyrene carrying amphiphilic PEGylated-perfluoroalkyl side chain (SmSzn) were synthesised by atom transfer radical polymerisation (ATRP) (2, Figure 2). ${ }^{[86]}$ Two main advantages of this architecture were a more precise control on its macromolecular structure and a better chemical compatibility with the SEBS matrix. The ATRP chemistry was tailored in order for the films to display well-defined surface morphologies (domain size 20-25 $\mathrm{nm}$ ), going from parallel lamellae to lying cylinders (Figure 4), up to spheres by changing the lengths ( $m$ and $n$ ) of the constituent blocks. Both X-ray photoelectron 
spectroscopy (XPS) and near-edge X-ray absorption fine structure (NEXAFS) analyses demonstrated that the 'dry' surfaces contained much more fluorine with respect to the theoretical composition. ${ }^{[88]}$ This was reduced in the 'wet' surfaces after water immersion due to the increased presence of hydrophilic PEG side chains at the polymer-water interface (Figure 5). Moreover, the hydrophobic phenyl rings migrated away from the surface after water immersion.
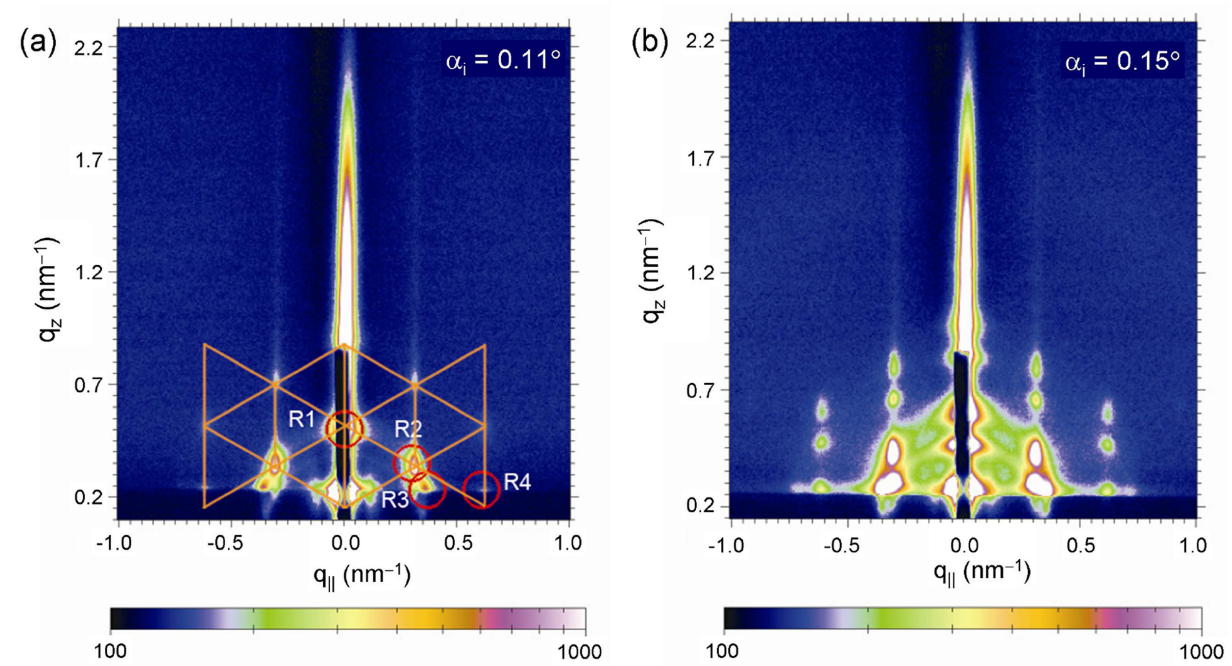

Figure 4. 2-Dimensional grazing-incidence small-angle X-ray scattering (GISAXS) maps of a block copolymer SmSzn $(m=51, n=17)$ acquired at an X-ray angle of (a) $0.11^{\circ}$ and (b) $0.15^{\circ}$. The hexagonal cylinder lattice is indicated in (a). At higher incident angles diffraction spots are split up due to reflection from the substrate. Reproduced from Ref. ${ }^{[86]}$ with permission of John Wiley and Sons.
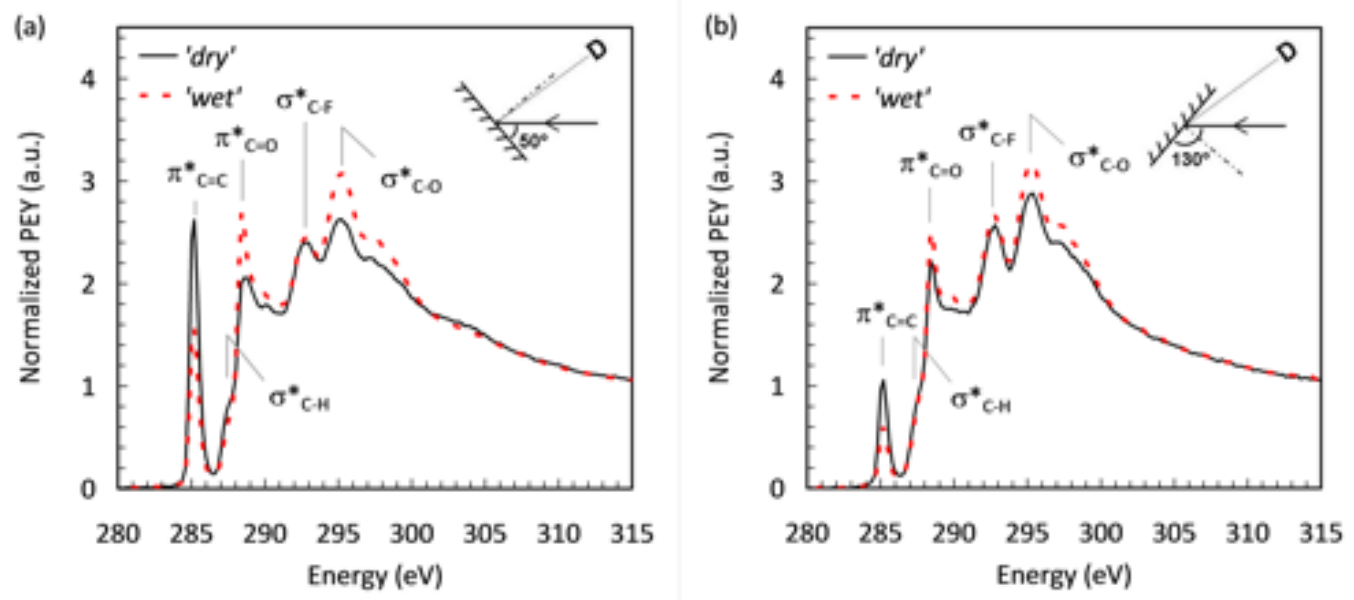

Figure 5. C(1s) NEXAFS spectra of 'dry' and 'wet' thin films of a block copolymer SmSzn $(m=51, n=17)$, acquired at X-ray incidence angles of (a) $50^{\circ}$ and (b) $130^{\circ}$. Note, e.g. the $\sigma^{*} \mathrm{C}-\mathrm{O}$ resonance for the PEG chains and the $\pi^{*}{ }_{\mathrm{C}=\mathrm{C}}$ resonance for the phenyl rings. Reproduced with permission from Ref. ${ }^{[88]}$. Copyright (2010) American Chemical Society. 